\title{
Quantum Phase Transition in the One-Dimensional XZ Model
}

\author{
W. BRZEZICKI ${ }^{a}$ AND A.M. OleŚ $S^{a, b}$ \\ ${ }^{a}$ M. Smoluchowski Institute of Physics, Jagiellonian University \\ Reymonta 4, PL-30-059 Kraków, Poland \\ ${ }^{b}$ Max-Planck-Institut für Festkörperforschung \\ Heisenbergstrasse 1, D-70569 Stuttgart, Germany
}

\begin{abstract}
We introduce a one-dimensional XZ model with alternating $\sigma_{i}^{z} \sigma_{i+1}^{z}$ and $\sigma_{i}^{x} \sigma_{i+1}^{x}$ interactions on even/odd bonds, interpolating between the Ising model and the quantum compass model. We present two ways of its exact solution by: (i) mapping to the quantum Ising models, and (ii) using fermions with spin $1 / 2$. In certain cases the nearest neighbor pseudospin correlations change discontinuously at the quantum phase transition, where one finds highly degenerate ground state of the 1D compass model.
\end{abstract}

PACS numbers: 75.10.Jm, 03.67.-a, 05.70.Fh

\section{Introduction}

Models of magnetism with exotic interactions are motivated by rather complex orbital superexchange in the Mott insulators. In certain case the degeneracy of $3 d$ orbitals is only partly lifted and the remaining orbital degrees of freedom are frequently described as $1 / 2$ spins. They also arise from spin-orbital superexchange, with rich dynamics leading to enhanced quantum fluctuations near quantum phase transitions [1], and to entangled spin-orbital ground states [2]. The orbital interactions have much lower symmetry than the $\mathrm{SU}(2)$ of spin interactions and their form depends on the orientation of the bond in real space [3], so they may lead to orbital liquid in three dimensions [4]. A generic and simplest model of this type is so-called compass model introduced in [5] when the coupling along a given bond is Ising-like, but different spin components are active along particular bonds, for instance $J_{x} \sigma_{i}^{x} \sigma_{j}^{x}$ and $J_{z} \sigma_{i}^{z} \sigma_{j}^{z}$ along $a$ and $b$ axis in the two-dimensional (2D) compass model [6]. This situation is quite different from classical Ising-like models with periodically distributed frustration [7].

Recently we discussed the properties of the one-dimensional (1D) model that interpolates between the Ising and compass model [8]. The solution based on specific choice of interactions indicated divergences in correlation functions while approaching the transition point. This suggested first-order phase transition but it was not clear if the effect is generic or it is just an artefact of singular parameterization of interactions. To answer this question we introduce more general solution which can be applied to any parameterization and then we make second insight into the original problem.

\section{2. $\mathrm{XZ}$ model in one dimension}

The model is described by a generalized XZ Hamiltonian with different energies for even and odd bonds, where the number of sites is $2 N$ and for simplicity we suppose that $N$ is even

$$
\begin{aligned}
\mathcal{H} & =\sum_{i=1}^{N}\left(J_{1} \sigma_{2 i-1}^{z} \sigma_{2 i}^{z}+J_{2} \sigma_{2 i-1}^{x} \sigma_{2 i}^{x}+L_{1} \sigma_{2 i}^{z} \sigma_{2 i+1}^{z}\right. \\
& \left.+L_{2} \sigma_{2 i}^{x} \sigma_{2 i+1}^{x}\right) .
\end{aligned}
$$

This Hamiltonian turns into the one discussed in Ref. [8] if we fix the energy constants as follows: $J_{1}(\alpha)=$ $\frac{1}{2}(|1-\alpha|+1-\alpha), \quad J_{2}(\alpha)=-J_{1}(\alpha)+1, L_{1}(\alpha)=$ $-J_{1}(-\alpha+2)+1, L_{2}(\alpha)=J_{1}(-\alpha+2)$, where $0 \leq \alpha \leq 2$. For $0 \leq \alpha \leq 1$ it gives

$$
\begin{aligned}
& \mathcal{H}(\alpha) \equiv \sum_{i=1}^{N}\left[(1-\alpha) \sigma_{2 i-1}^{z} \sigma_{2 i}^{z}+\alpha \sigma_{2 i-1}^{x} \sigma_{2 i}^{x}\right. \\
& \left.\quad+\sigma_{2 i}^{z} \sigma_{2 i+1}^{z}\right],
\end{aligned}
$$

and for $1<\alpha \leq 2$ we transform $\sigma_{i}^{x} \leftrightarrow \sigma_{i}^{z},\{2 i-1,2 i\} \leftrightarrow$ $\{2 i, 2 i+1\}$ for all $i$ and $\alpha \rightarrow(2-\alpha)$.

\section{First solution}

To solve the model given by Eq. (2) we choose eigenbasis of $\sigma_{i}^{z}$ operators consisting of vectors $\left|s_{1}, s_{2}, s_{3}, \ldots, s_{2 N}\right\rangle$ with $s_{i}= \pm 1$ for all $i$. Every state like that can be denoted equivalently as

$$
\begin{aligned}
& \left|t_{1}, t_{2}, \ldots, t_{N}\right\rangle_{r_{1} r_{2} \ldots r_{N}} \equiv \mid t_{1}, t_{1} r_{1}, t_{2}, t_{2} r_{2}, \ldots, \\
& \left.\quad t_{N}, t_{N} r_{N}\right\rangle
\end{aligned}
$$

where $t_{i} \equiv s_{2 i-1}$ and $r_{i} \equiv s_{2 i-1} s_{2 i}$ for $i=1,2, \ldots, N$. This will let us exploit the fact that the Hamiltonian (2) 
flips only odd pairs of spins. For states like (3), we define new spin operators $\tau_{i}^{\prime} z$ and $\tau_{i}^{x}$ which act only on $t_{1}, t_{2}, \ldots, t_{N}$ quantum numbers

$$
\begin{aligned}
& \tau_{1}^{x}\left|t_{1}, t_{2}, \ldots, t_{N}\right\rangle_{r_{1} r_{2} \cdots r_{N}}=\left|-t_{1}, t_{2}, \ldots, t_{N}\right\rangle_{r_{1} r_{2} \cdots r_{N}}, \\
& \tau_{1}^{\prime}{ }^{z}\left|t_{1}, t_{2}, \ldots, t_{N}\right\rangle_{r_{1} r_{2} \cdots r_{N}}=t_{1}\left|t_{1}, t_{2}, \ldots, t_{N}\right\rangle_{r_{1} r_{2} \cdots r_{N}} .
\end{aligned}
$$

Next we transform each $\tau_{i}^{\prime z}$ as follows $\tau_{i}^{\prime} z \equiv$ $r_{1} r_{2} \cdots r_{i-1} \tau_{i}^{z}$, to get effective forms of the Hamiltonian in subspaces spanned by vectors (3) with fixed $r_{i}$ 's

$$
\mathcal{H}_{r_{1} r_{2} \cdots r_{N}}(\alpha)=\sum_{i=1}^{N}\left(\tau_{i}^{z} \tau_{i+1}^{z}+\alpha \tau_{i}^{x}\right)+C_{\boldsymbol{r}}(\alpha),
$$

where $\tau_{N+1}^{z}=r_{1} r_{2} \ldots r_{N} \tau_{1}^{z}$ and $C_{\boldsymbol{r}}(\alpha)=(1-\alpha) \sum_{i=1}^{N} r_{i}$. One finds the quantum Ising model (QIM) which may be exactly solved [9]; here we solve it either with periodic or antiperiodic boundary condition. The solution was described in detail in Ref. [8]. First step is to introduce the Jordan-Wigner (JW) transformation,

$$
\tau_{j}^{z}=\left(c_{j}+c_{j}^{\dagger}\right) \prod_{i<j}\left(1-2 c_{i}^{\dagger} c_{i}\right), \quad \tau_{j}^{x}=1-2 c_{j}^{\dagger} c_{j} .
$$

The boundary condition for fermion operators $\left\{c_{i}\right\}$ differs for even and odd number of quasiparticles in the chain. Fortunately, the Hamiltonian conserves the parity of fermions, this lets us write $\mathcal{H}_{\boldsymbol{r}}=\frac{1}{2}(1+P) \mathcal{H}_{\boldsymbol{r}}^{+}+$ $\frac{1}{2}(1-P) \mathcal{H}_{\boldsymbol{r}}^{-}$, where

$$
\begin{aligned}
& \mathcal{H}_{\boldsymbol{r}}^{ \pm}=\sum_{i=1}^{N}\left[\left(c_{i}^{\dagger}-c_{i}\right)\left(c_{i+1}^{\dagger}-c_{i+1}\right)-2 \alpha c_{i}^{\dagger} c_{i}\right] \\
& +C_{\boldsymbol{r}}(\alpha),
\end{aligned}
$$

and where $\frac{1}{2}(1 \pm P)$ are projections on subspaces with even $(+)$ and odd $(-)$ number of quasiparticles. Here we adopt notation $\boldsymbol{r}$ for the subspace labels $r_{1} r_{2} \ldots r_{N}$. The boundary conditions are $c_{N+1}=\mp c_{1} \prod_{i=1}^{N} r_{i}$ for $\mathcal{H}_{\boldsymbol{r}}^{ \pm}$, respectively. After the Fourier transformation, $c_{j}=$ $\frac{1}{\sqrt{N}} \sum_{k}{ }^{ \pm} \mathrm{e}^{\mathrm{i} j k} c_{k}$ we find (7) in a block diagonal form

$$
\begin{aligned}
& \mathcal{H}_{\boldsymbol{r}}^{ \pm}=\sum_{k}^{\boldsymbol{r} \pm}\left[c_{k}^{\dagger} c_{k}(2 \cos k-2 \alpha)+\left(c_{k}^{\dagger} c_{-k}^{\dagger} \mathrm{e}^{\mathrm{i} k}+\text { h.c. }\right)\right] \\
& \quad+C_{\boldsymbol{r}}(\alpha)+N \alpha,
\end{aligned}
$$

where quasimomenta $k$ take "integer" values $k=$ $0, \pm \frac{2 \pi}{N}, \pm 2 \frac{2 \pi}{N}, \ldots, \pi$ for a periodic boundary condition and in the antiperiodic case they are "half-integer" $k=$ $0, \pm \frac{2 \pi}{N}, \pm 2 \frac{2 \pi}{N}, \ldots, \pi$. Diagonalization is completed by a Bogoliubov transformation $\gamma_{k}^{\dagger}=u_{k} c_{k}^{\dagger}+v_{k} c_{-k}$ performed for all $k>0$ and $k \neq \pi$ where $\left(u_{k}, v_{k}\right)$ are eigenmodes of the Bogoliubov-de Gennes equation $\left[\mathcal{H}_{r}^{ \pm}, \gamma_{k}^{\dagger}\right]=E_{k} \gamma_{k}^{\dagger}$. In this way we get the full Hamiltonian spectrum in every subspace $\boldsymbol{r}$. For instance, in case when $\prod_{i=1}^{N} r_{i}=1$ we obtain a Hamiltonian for even number of quasiparticles $\gamma_{k}$ :

$$
\mathcal{H}_{\boldsymbol{r}}^{+}(\alpha)=\sum_{k}^{r+} E_{k}\left(\gamma_{k}^{\dagger} \gamma_{k}-\frac{1}{2}\right)+C_{\boldsymbol{r}}(\alpha),
$$

where $E_{k}=2\left(1+\alpha^{2}-2 \alpha \cos k\right)^{1 / 2}$ is the quasiparticle energy. The ground state is the Bogoliubov vacuum in a subspace where all $r_{i}=-1$, apart from points $\alpha=0,1,2$ it has no degeneracy. Cases of $\alpha=1,2$ are trivial. For $\alpha=1$ we have $C_{\boldsymbol{r}}(\alpha)=0$ for all $\boldsymbol{r}$ which means that there is a ground state in every subspace where $\prod_{i=1}^{N} r_{i}=1$. This results in $2^{N-1}$-fold degeneracy for the $1 \mathrm{D}$ compass model (Fig. 1a). In the limit $N \rightarrow \infty$ the lowest energies of periodic and antiperiodic QIM get equal at $\alpha=1$. For $0<\alpha \leq 1$ they are already two- and threefold degenerate, so when $\alpha=1$ the total degeneracy is $5 \times 2^{N}$, and the spin gap vanishes [8].

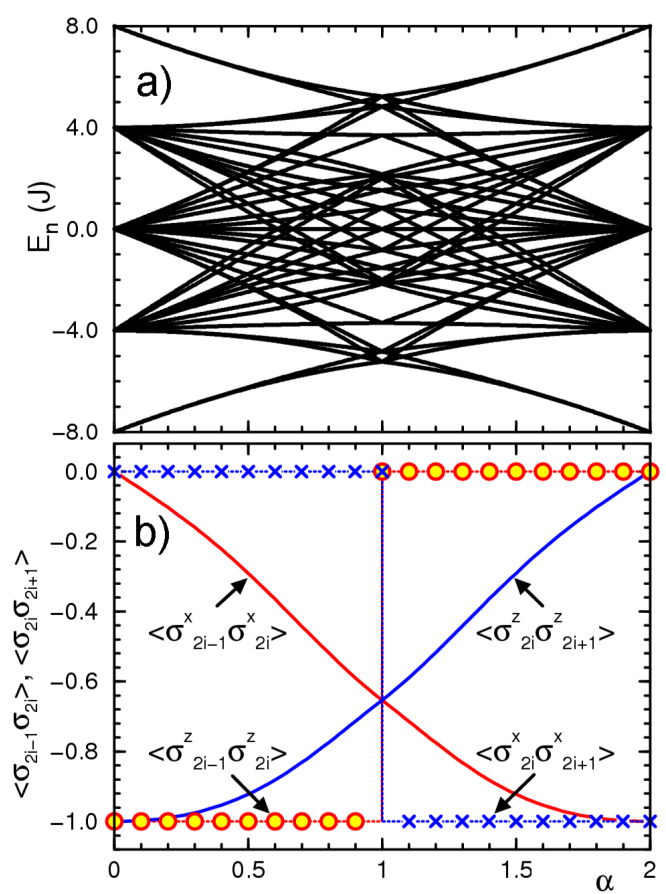

Fig. 1. (a) Eigenenergies $E_{n}$ of the XX-ZZ model (2) for $2 N=8$ sites and for increasing $\alpha$. Level crossing at $\alpha=1$ marks the quantum critical point of the 1D compass model. (b) Intersite pseudospin correlations on odd $\{2 i-1,2 i\}$ and even $\{2 i, 2 i+1\}$ bonds in the XX-ZZ model (2) for increasing $\alpha$. A transition between two types of pseudo-order, with $\left\langle\sigma_{2 i-1}^{z} \sigma_{2 i}^{z}\right\rangle=-1$ for $\alpha<1$ and $\left\langle\sigma_{2 i}^{x} \sigma_{2 i+1}^{x}\right\rangle=-1$ for $\alpha>1$, occurs at the quantum critical point $\alpha=1$, where only $\left\langle\sigma_{2 i-1}^{x} \sigma_{2 i}^{x}\right\rangle=\left\langle\sigma_{2 i}^{z} \sigma_{2 i+1}^{z}\right\rangle=-\frac{2}{\pi}$ are finite. These results are reproduced from Ref. [8].

\section{Second solution}

The most direct way of dealing with Eq. (1) is to leave the interactions $J_{1,2}$ and $L_{1,2}$ undefined and to start with the JW transformation

$$
\begin{aligned}
\sigma_{j}^{z} & =\left(c_{j}+c_{j}^{\dagger}\right) \prod_{i<j}\left(1-2 c_{i}^{\dagger} c_{i}\right), \\
\sigma_{j}^{x} & =\frac{1}{i}\left(c_{j}-c_{j}^{\dagger}\right) \prod_{i<j}\left(1-2 c_{i}^{\dagger} c_{i}\right),
\end{aligned}
$$

which transforms spins into fermion operators $c_{j}$. Here 
the crucial step is to introduce new quantum number for fermions with two possible values $n$ and $p$. This can be regarded as quasiparticles' spin or as splitting the chain into bi-atomic elementary cells. We define $c_{i}^{n} \equiv c_{2 i-1}$ and $c_{i}^{p} \equiv c_{2 i}$. Because of the boundary conditions and the fact that $\mathcal{H}$ preserves the parity of fermions, we obtain

$$
\begin{aligned}
\mathcal{H}^{ \pm} & =\sum_{i=1}^{N}\left(J^{+} c_{i}^{n \dagger} c_{i}^{p}+L^{+} c_{i}^{p \dagger} c_{i+1}^{n}+J^{-} c_{i}^{n \dagger} c_{i}^{p \dagger}\right. \\
& \left.+L^{-} c_{i}^{p \dagger} c_{i+1}^{n \dagger}+\text { h.c. }\right)
\end{aligned}
$$

where $\mathcal{H}^{+}\left(\mathcal{H}^{-}\right)$is defined in subspace with even (odd) number of fermions with $c_{N+1}^{n}=-c_{1}^{n}\left(c_{N+1}^{n}=c_{1}^{n}\right)$. Here we introduced new notation for $J^{\prime}$ 's; $J^{ \pm}=J_{1} \pm J_{2}$ and analogically for $L$ 's. Now, we proceed with the Fourier transform $c_{j}^{n, p}=\frac{1}{\sqrt{N}} \sum_{k}{ }^{ \pm} \mathrm{e}^{i j k} c_{k}^{n, p}$ for $n$ and $p$ fermions separately, compatible to fermions' parity. For periodic boundary conditions $k$ 's take "integer" values and in the antiperiodic case they are "half-integer". Finally, we find the problem block diagonal in a form

$$
\begin{gathered}
\mathcal{H}^{+}=\sum_{k}^{+}\left[c_{k}^{n \dagger} c_{-k}^{p \dagger}\left(J^{-}-L^{-} \mathrm{e}^{-\mathrm{i} k}\right)\right. \\
\left.+c_{k}^{n \dagger} c_{k}^{p}\left(J^{+}+L^{+} \mathrm{e}^{-\mathrm{i} k}\right)+\text { h.c. }\right]
\end{gathered}
$$

and for $\mathcal{H}^{-}$similarly but with $k=0, \pi$ possible. Diagonalization is completed by a four-dimensional Bogoliubov transformation. We search for invariant subspace of a linear operator $\left[\mathcal{H}^{+},.\right]$in eight-dimensional space spanned by $c_{ \pm k}^{n(\dagger)}$ and $c_{ \pm k}^{p(\dagger)}$. The result suggests the form of transformation as

$$
\begin{aligned}
& \left(\begin{array}{c}
\gamma_{k}^{n \dagger} \\
\gamma_{-k}^{n} \\
\gamma_{k}^{p \dagger} \\
\gamma_{-k}^{p}
\end{array}\right)=\hat{\beta}_{k}\left(\begin{array}{c}
c_{k}^{n \dagger} \\
c_{-k}^{n} \\
c_{k}^{p \dagger} \\
c_{-k}^{p}
\end{array}\right) \quad(0 \leq k<\pi), \\
& \left(\begin{array}{c}
\gamma_{\pi}^{n \dagger} \\
\gamma_{\pi}^{p \dagger}
\end{array}\right)=\hat{\beta}_{\pi}\left(\begin{array}{c}
c_{\pi}^{n \dagger} \\
c_{\pi}^{p \dagger}
\end{array}\right),
\end{aligned}
$$

where $\hat{\beta}_{k}$ and $\hat{\beta}_{\pi}$ are orthogonal matrices. This assures that $\gamma_{k}$ 's are fermionic. The rows of $\hat{\beta}$ are eigenvectors of the Bogoliubov-de Gennes equation $\left[\mathcal{H}, \gamma_{k}^{n \dagger}\right]=E_{k} \gamma_{k}^{n \dagger}$ and eigenvalues $E_{k}$ are energies of system's elementary excitations. Finally, we find $\mathcal{H}^{+}$in a diagonal form

$$
\begin{gathered}
\mathcal{H}^{+}=\sum_{k}^{+}\left[E_{k}^{n}\left(\gamma_{k}^{n \dagger} \gamma_{k}^{n}-\frac{1}{2}\right)\right. \\
\left.+E_{k}^{p}\left(\gamma_{k}^{p \dagger} \gamma_{k}^{p}-\frac{1}{2}\right)\right],
\end{gathered}
$$

where $E_{k}^{n}=2\left(J_{1}^{2}+L_{2}^{2}+2 J_{1} L_{2} \cos k\right)^{1 / 2}, E_{k}^{p}=2\left(J_{2}^{2}+\right.$ $\left.L_{1}^{2}+2 J_{2} L_{1} \cos k\right)^{1 / 2}$. In a similar way we get the result for $\mathcal{H}^{-}$. Luckily, the parity of particles $\gamma_{k}$ is the same as parity of original JW fermions, thus only states with even (odd) number of quasiparticles $\gamma_{k}$ belong to the spectrum of $\mathcal{H}^{+}\left(\mathcal{H}^{-}\right)$. The ground state energy $E_{0}$ obtained from (14) is $E_{0}=-\frac{1}{2} \sum_{k}{ }^{+}\left[E_{k}^{n}+E_{k}^{p}\right]$. Putting $J_{1,2}=J_{1,2}(\alpha)$ and $L_{1,2}=L_{1,2}(\alpha)$ we find the same energy spectrum as described in Ref. [8] (see Fig. 1a). For $\alpha \leq 1$ one finds $E_{k}^{n}=2(1-\alpha)$ and $E_{k}^{p}=\left\{1+\alpha^{2}-2 \alpha \cos k\right\}^{1 / 2}$, which means that the occupation numbers $\gamma_{k}^{n \dagger} \gamma_{k}^{n}$ play the role of the subspace indexes $r_{i}$ from the previous solution while $\gamma_{k}^{p \dagger} \gamma_{k}^{p}$ describe excitations within a given subspace.

Pseudospin correlation functions can be derived from $E_{0}$ as derivatives with respect to $J_{1,2}$ and $L_{1,2}$, respectively. The main result is that $\left\langle\sigma_{2 i-1}^{z} \sigma_{2 i}^{z}\right\rangle$ and $\left\langle\sigma_{2 i}^{x} \sigma_{2 i+1}^{x}\right\rangle$ remain constant in intervals $[0,1)$ and $(1,2]$, but with discontinuities at $\alpha=1$ (see Fig. 1b). The origin of these singularities is a cusp of $E_{k}^{n}\left(J_{1}, L_{2}\right)$ surface at $\left(J_{1}, L_{2}\right)=$ $(0,0)$. The trajectory $\left(J_{1}, L_{2}\right)(\alpha)$ passes through this point at $\alpha=1$. This means that no first order phase transition occurs in the general model of Eq. (1), unless curves $\left(J_{1}, L_{2}\right)(\alpha)$ or $\left(J_{2}, L_{1}\right)(\alpha)$ pass through $(0,0)$. Passing should be interpreted literally as passing, not reversing at $(0,0)$. For example, the curve $(x, y)=\left((\alpha-1)^{2},(\alpha-1)^{3}\right)$ reaches the point $(0,0)$ at $\alpha=1$, but nevertheless one of the pseudospin correlations remains continuous.

\section{Summary}

We have presented an exact solution which demonstrated that a hidden order with constant pseudospin correlations exists in the $1 \mathrm{D}$ XZ model. The second method used for solving this problem provides more insight into the nature of the quantum phase transition as discussed in Ref. [8], while the first one is more flexible and may be generalized, for instance, to the ladder geometry.

\section{Acknowledgments}

We acknowledge support by the Foundation for Polish Science (FNP). This work was supported by the Polish Ministry of Science and Higher Education under project No. N202 068 32/1481.

\section{References}

[1] L.F. Feiner, A.M. Oleś, J. Zaanen, Phys. Rev. Lett. 78, 2799 (1997).

[2] A.M. Oleś, P. Horsch, L.F. Feiner, G. Khaliullin, Phys. Rev. Lett. 96, 147205 (2006).

[3] J. van den Brink, New J. Phys. 6, 201 (2004).

[4] G. Khaliullin, S. Maekawa, Phys. Rev. Lett. 85, 3950 (2000).

[5] K.I. Kugel, D.I. Khomskii, Sov. Phys. Usp. 25, 231 (1982).

[6] J. Dorier, F. Becca, F. Mila, Phys. Rev. B 72, 024448 (2005).

[7] L. Longa, A.M. Oleś, J. Phys. A 13, 1031 (1980).

[8] W. Brzezicki, J. Dziarmaga, A.M. Oleś, Phys. Rev. B 75, 134415 (2007).

[9] J.H.H. Perk, H.W. Campel, G.R.W. Quispel, F.W. Nijhoff, Physica A 123, 1 (1984). 